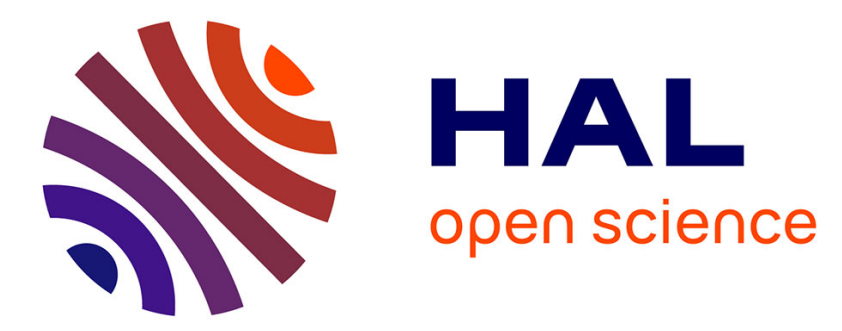

\title{
Local structure of polymeric ferrogels
}

José Alberto Galicia, Fabrice Cousin, Emmanuelle Dubois, Valérie Cabuil, Olivier Sandre, Régine Perzynski

\section{To cite this version:}

José Alberto Galicia, Fabrice Cousin, Emmanuelle Dubois, Valérie Cabuil, Olivier Sandre, et al.. Local structure of polymeric ferrogels. Journal of Magnetism and Magnetic Materials, 2010, 323 (10), pp.1211-1215. 10.1016/j.jmmm.2010.11.008 . hal-02169248

\section{HAL Id: hal-02169248 \\ https://hal.science/hal-02169248}

Submitted on 31 May 2020

HAL is a multi-disciplinary open access archive for the deposit and dissemination of scientific research documents, whether they are published or not. The documents may come from teaching and research institutions in France or abroad, or from public or private research centers.
L'archive ouverte pluridisciplinaire HAL, est destinée au dépôt et à la diffusion de documents scientifiques de niveau recherche, publiés ou non, émanant des établissements d'enseignement et de recherche français ou étrangers, des laboratoires publics ou privés. 


\title{
Journal of Magnetism and Magnetic Materials
}

Journal of Magnetism and Magnetic Materials 323 (2011) 1211-1215

\section{Local structure of polymeric ferrogels}

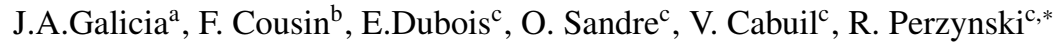 \\ ${ }^{a}$ Fac. de Ing.Quimica, Benemerita Univ. Autonoma de Puebla, Edif 1471102 Ciudad Universitaria, 18 sur y San Claudio, Puebla, Pue, Mexico \\ ${ }^{b}$ Lab. Léon Brillouin, UMR 12 CNRS-CEA, CEA-Saclay, 91191 Gif-sur-Yvette, France \\ ${ }^{c}$ UPMC Univ. Paris 6, Laboratoire PECSA (UMR 7195 UPMC-CNRS-ESPCI), Case 514 Place Jussieu, F-75005 Paris FRANCE
}

Author manuscript version of article available in its final form on the publisher's website: DOI 10.1016/j.jmmm.2010.11.008

\begin{abstract}
We synthesize hybrid gels incorporating $\gamma-\mathrm{Fe}_{2} \mathrm{O}_{3}$ nanoparticles (NP's), citrate coated, in a polyacrylamide (PAM) network. The local organisation and the rotational degrees of magnetic nanoparticles are probed in the conditions of gel synthesis and also at swelling equilibrium, to correlate the homogeneous/ inhomogeneous structure of the ferrogels to the synthesis parameters and to their macroscopic elasticity. NP adsorption on the PAM network at low citrate concentration is responsible for the reinforcing of the polymer structure. At higher citrate concentration, due to a competition between citrate and NP's, the nanoparticles desorb from the polymer structure weakening the system.
\end{abstract}

PACS 82.35.NP; 61.05.fg; 78.20.Ls

Keywords: Nanoparticles in polymers; Neutron scattering; Magneto-optical effect

\section{Introduction}

Polymeric ferrogels are organic/inorganic composites prepared for ferroelastic applications. They are based on the intimate mixing of polymeric gels and magnetic particles $[1,2]$. Frequently, in order to mechanically reinforce the polymeric structure, the particles are aggregated $[3,4,5]$. The swelling of the system is then seriously hindered. Our aim in this work is to understand how to reinforce homogeneously a polymeric gel with nanoparticles (NPs), keeping as far as possible the swelling properties of the gel. We choose to use here a versatile model-system combining - the well-known poly(acrylamide) (PAM) polymeric system and - magnetic NPs as fillers, in order to also use these NPs as local probes of the system. Our objective is to point out the problems and to determine the relevant parameters for a homogeneous/inhomogeneous NP incorporation reinforcing the polymer structure. We associate two

\footnotetext{
${ }^{*}$ Corresponding author. Tel.: +33-1-444273267; fax: +33-1444273228.

Email address: regine.perzynski@upmc.fr (R. Perzynski)
}

experimental techniques, a static probing of the NP distribution in the ferrogel by Small Angle Neutron Scattering (SANS) with a dynamic probing of the NP rotational degrees of freedom by relaxation of magnetooptical birefringence.

\section{Experimental}

PAM networks are well known for their swelling properties and worldly used as superabsorbing microbeads or as separation media in biochemistry for electrophoresis. The gels are here obtained by polymerizing acrylamide (AM) monomers either in aqueous medium (for hydrogels) or in a colloidal suspension of citrate-coated maghemite NPs (for ferrogels) in the presence of the cross-linking agent $N, N^{\prime}$-methylenebis-acrylamide (BAM). Hydrogels and ferrogels are out-of-equilibrium systems in their synthesis conditions with a mesh size $\xi_{\text {synth }}$, which is strongly dependent on $C L=\mathrm{BAM} / \mathrm{AM}$ the cross-linking ratio of the system, here $0.5 \% \leqslant C L \leqslant 2 \%$ in order to have homogeneous hydrogels [6]. Put in a water bath (at the same 
ionic strength as the synthesis medium) they swell by absorbing water, reaching swelling equilibrium in typically three weeks. In the case of pure PAM hydrogel at

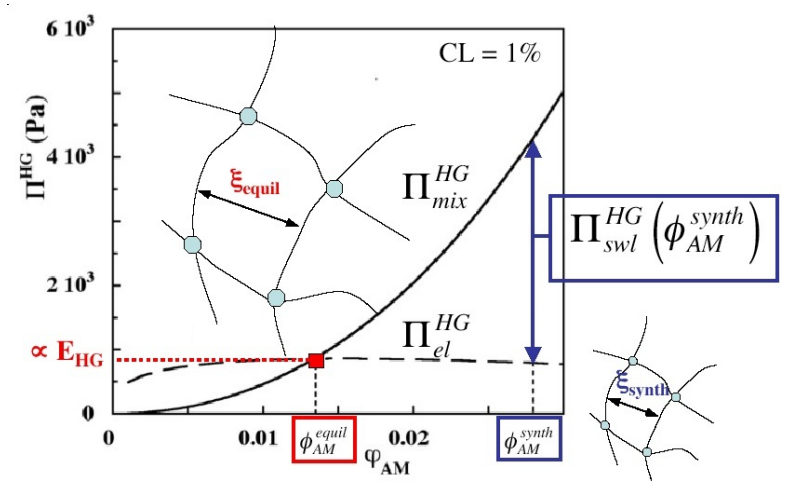

Figure 1: Mixing (full line) and elastic (dashed line) osmotic pressure of hydrogels at $C L=1 \%$ as a function of the volume fraction $\phi_{A M}$ of AM. The polymeric array is sketched in synthesis and swelling conditions

$C L=1 \%$, Figure 1 illustrates the behavior of its mixing $\Pi_{m i x}^{H G}$ and of its elastic $\Pi_{e l}^{H G}$ osmotic pressures as a function of the volume fraction $\phi_{A M}$ of $A M$. During the swelling process, the mesh of the polymeric array evolves from $\xi_{\text {synth }}$ towards $\xi_{\text {equil }}$ and at swelling equilibrium $\Pi_{m i x}^{H G}=\Pi_{e l}^{H G}$, their value being proportional to the Young modulus $E_{H G}$ of the hydrogel. On the contrary in the synthesis conditions, $\Pi_{m i x}^{H G}$ and $\Pi_{e l}^{H G}$ differ by an osmotic pressure $\Pi_{s w l}^{H G}$.

While synthesizing ferrogels, $\Pi_{s w l}^{H G}$ has to be compatible with the NP osmotic pressure $\Pi^{M L}$ in the magnetic liquid (ML) used for the synthesis. Basically $\Pi^{M L}$ depends on - the NP volume fraction $\Phi$, - the NP diameter $d$ and - the ML ionic strength [7].

Two parameters are thus important to consider in the synthesis conditions : the geometrical ratio $R_{S}=d^{3} / \xi_{\text {synth }}^{3}$ and the osmotic ratio $R_{\Pi}=$ $\Pi^{M L} / \Pi_{s w l}^{H G}$. We show below how they tune the homogeneity/inhomogeneity of the composite. They depend on the following synthesis parameters : $-C L$ and $\phi_{A M}^{\text {synth }}$ for the polymeric solution and - $d, \Phi_{\text {synth }}$ and $[\text { cit }]_{\text {free }}$ the concentration of free citrate species for the ML.

\subsection{Experimental conditions}

The ferrogels are prepared as described in [9] at $C L$ $=0.5 \%, 1 \%$ or $2 \%$, with ML at $p H=7.2$, based on $\gamma$ $\mathrm{Fe}_{2} \mathrm{O}_{3}$ NPs synthesized as in [8], stabilized by a citrate coating, with $d$ of the order of $10 \mathrm{~nm}$. The osmotic compression technique allows us to precisely fix $-\Phi_{\text {synth }}$ between $1 \%$ and $9 \%$ and $-[\mathrm{cit}]_{\text {free }}$ in synthesis conditions and also at swelling equilibrium (here $[\text { cit }]_{\text {free }}=810^{-3}$ mol.L $\mathrm{L}^{-1}$ or 0.1 mol.L $\mathrm{L}^{-1}$ in both cases) [7].

Attempts to prepare ferrogels at $R_{\Pi}$ larger than 1 have led to samples macroscopically inhomogeneous.

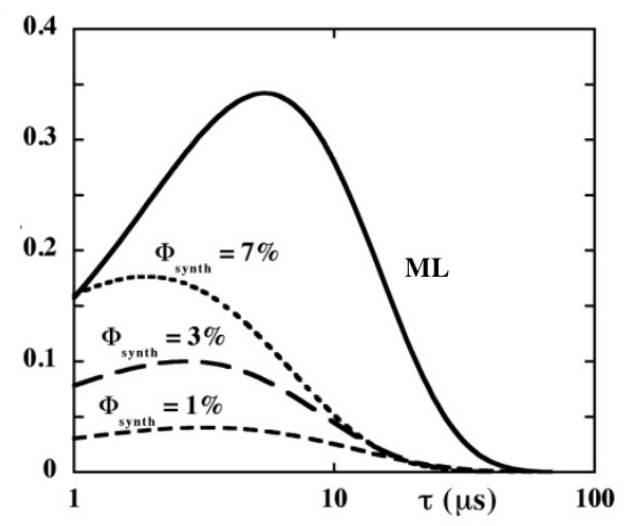

Figure 2: Normalized distributions of NP rotational times - in the initial ML and - in ferrogels in synthesis conditions at various $\Phi_{\text {synth }}$ $\left([\text { cit }]_{\text {free }}=810^{-3} \mathrm{~mol} . \mathrm{L}^{-1}\right)$. The proportion of rotating NPs increases as $\Phi_{\text {synth }}$ increases.

\subsection{Measurements}

We develop several measurements in order to answer to the following questions. How varies the local distribution of NPs in the ferrogels? Is the distribution homogeneous or are there local pockets of magnetic liquid ? Are the NPs free to rotate or are they decorating the polymeric chains ? What happens during the swelling process ? Is there any release of NP ?

We thus determine the swelling ratios $G_{\text {synth }}$ in the synthesis conditions and $G_{\text {equil }}$ at swelling equilibrium by weight measurements as $G_{\text {synth,equil }}=\left(m_{F G}-\right.$ $\left.m_{d r y}\right) /\left(m_{d r y}-m_{N P}\right)$ and also the ratio $R$ of NP release as $R=\left(m_{\text {part }}^{\text {synth }}-m_{\text {part }}^{\text {equil }}\right) / m_{\text {part }}^{\text {synth }}$.

It is checked that $\Phi_{\text {equil }}=\Phi_{\text {synth }}(1-R) G_{\text {synth }} / G_{\text {equil }} \leqslant$ $\Phi_{\text {synth }}$ by determination of the NP volume fractions $\Phi_{\text {synth }}$ and $\Phi_{\text {equil }}$ with iron titration using Atomic Emission Spectroscopy.

NP structure factors are determined by SANS in the initial magnetic liquids and in the ferrogels at swelling equilibrium, at LLB facility (CEA-Saclay, France) on PAXY spectrometer for wave-vectors $0.0065 \AA^{-1} \leqslant$ $q \leqslant 0.15 \AA^{-1}[10]$. In the ferrogels, the neutron scattering contrast of the solvent is adjusted to match that of the PAM replacing water by a $44 / 56 \% \mathrm{H}_{2} \mathrm{O} / \mathrm{D}_{2} \mathrm{O}$ mixture. In these conditions the nuclear contribution of the NP largely dominates their magnetic contribution [11]. Thanks to the NP magneto-optical properties, 


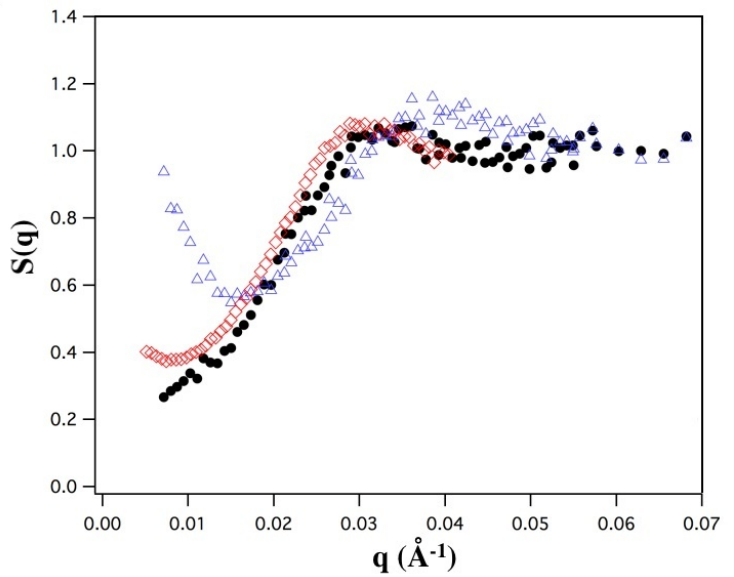

Figure 3: NP structure factor $\mathrm{S}(\mathrm{q})$ in the initial ML at $\Phi=3 \%$ (black dots) and in ferrogels at swelling equilibrium : (red open circles) $\Phi_{\text {equil }}=3 \%$ synthesized at $\Phi_{\text {synth }}=7 \%, C L=0.5 \%, R_{S}<1$ and $R_{\Pi}<1$ (homogeneous ferrogel); (blue triangles) $\Phi_{\text {equil }}=0.76 \%$, synthesized at $\Phi_{\text {synth }}=3 \%, C L=2 \%, R_{S} \sim 1$ and $R_{\Pi}<1$ (heterogeneous ferrogel - see ahead Table 1 - Series I); Here $[\text { cit }]_{\text {free }}=810^{-3}$ mol. $\mathrm{L}^{-1}$.

we measure by magneto-birefringence the proportion of NPs free to rotate in the ferrogels and their distribution of characteristic rotational time, in synthesis conditions and at swelling equilibrium $[10,12]$.

\section{Results and discussion at low $[\text { cit }]_{\text {free }}$}

We first study systems at $[\text { cit }]_{\text {free }}=8 \quad 10^{-3}$ mol. $\mathrm{L}^{-1}$ both in synthesis conditions and at swelling equilibrium.

\subsection{Experimental results at $R_{S}<1$ and $R_{\Pi}<1$}

In the synthesis conditions, a large quantity of NPs are blocked in rotation as attested by the distributions of NP rotating times presented in Figure 2, deduced from the measurements of magneto-birefringence and normalized in order to directly show the proportion of rotating NPs with respect to the initial ML. The proportion of blocked NPs varies with $\Phi_{\text {synth }}$.

At swelling equilibrium, we observe that the NP release $R$ is important and that it is smoothly increasing with $\Phi_{\text {synth }}$ up to a saturation at $R$ of the order of $50 \%$ for $\Phi_{\text {synth }}$ larger than $4 \%$. The swelling ratios $G_{\text {equil }}^{F G}$ are larger than $G_{s y n t h}^{H G}$ and always smaller than $G_{\text {equil }}^{H G}$ meaning that the NPs partly hinder the swelling of the polymeric chains.

The NP volume fraction $\Phi_{\text {equil }}$ remaining in the ferrogels at swelling equilibrium roughly equals that of blocked NPs in the synthesis conditions. Magnetobirefringence shows that almost all these NPs remaining in the ferrogel at swelling equilibrium, are also blocked in rotation, while the SANS measurements give structure factors $S(q)$ close to those of pure MLs at $\Phi \sim \Phi_{\text {equil }}$. In Figure 3, the structure factor of the ferrogel synthesized at $\Phi_{\text {synth }}=7 \%$ and $R_{S}<1$ presents in swelling conditions a maximum at $q_{\max }=0.032 \AA^{-1}$, corresponding to a most probable interparticle distance $\delta_{m p} \sim 200 \AA$, of the same order as the mesh size $\xi_{\text {equil }}=$ $170 \AA$ of the polymeric array and as the mean interparticle distance $\delta_{\text {mean }}$ deduced from the value $\Phi_{\text {equil }}=3 \%$ of the ferrogel. It demonstrates that the NP dispersions in the swollen ferrogels are rather homogeneous with roughly one NP per mesh of the polymeric array. These NPs being blocked in rotation [10], they homogeneously decorate the polymeric array and reinforce the network as can be attested by a mechanical probing of the elastic properties of these swollen ferrogels [9]. Figure 4 shows the relative variation of Young modulus $E_{F G}$ of swollen ferrogels at $C L=1 \%, R_{S}<1$ and $R_{\Pi}<1$ as a function of the ratio $\Phi_{\text {equil }} / \phi_{A M}$. The NPs increase here the Young modulus up to a factor 10. It can be adjusted by: $E_{F G}=E_{H G}\left(1+0.1 e^{3.8 \Phi_{\text {equil }} / \phi_{A M}}\right)$.

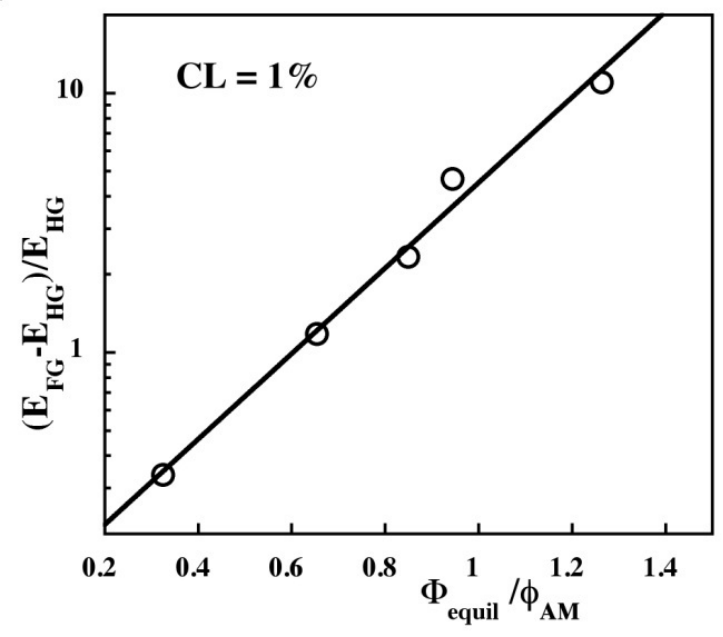

Figure 4: Semi-logarithmic representation of $\left(E_{F G}-E_{H G}\right) / E_{H G}$ as a function of the ratio $\Phi_{\text {equil }} / \phi_{A M}, E_{F G}$ is the Young modulus of swollen ferrogels at $C L=1 \%, R_{S}<1$ and $R_{\Pi}<1$ and $E_{H G}$ the Young modulus of the corresponding hydrogel, here $[\text { cit }]_{\text {free }}=810^{-3}$ mol.L $\mathrm{L}^{-1}$. The full line is a best fit of the data (see text).

Figure 5 compares the volume ratio of blocked nanoparticles inside the ferrogel before and after the swelling process as a function of $\Phi_{\text {synth }}$. These blocked NP's are adsorbed on the polymeric network in the synthesis conditions and remain adsorbed at swelling equi- 


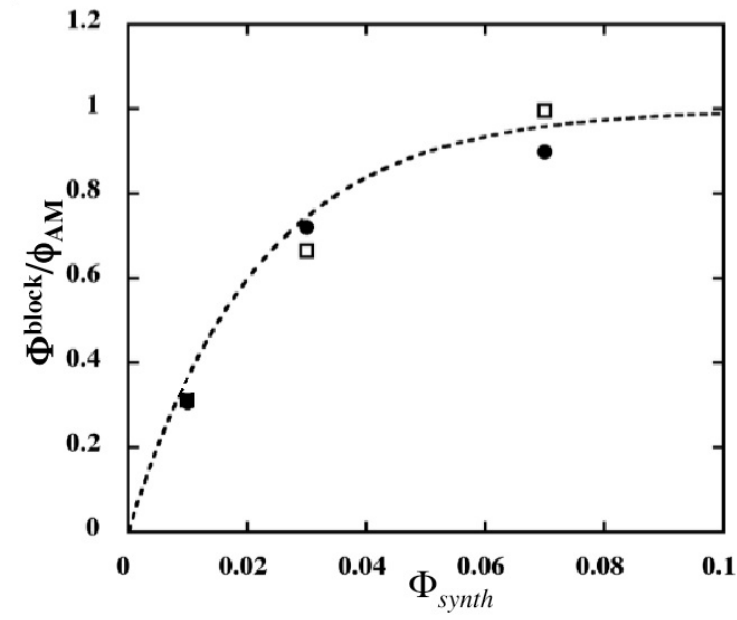

Figure 5: Nanoparticle adsorption in homogeneously decorated networks ([cit $]_{\text {free }}=810^{-3}$ mol.L ${ }^{-1}, C L=0.5 \%, R_{S}<1$ and $\left.R_{\Pi}<1\right)$; Volume ratio of blocked NP's with respect to polymer - in the synthesis conditions (closed circles) and - at swelling equilibrium (open squares) versus initial volume fraction of nanopaticles $\Phi_{\text {synth }}$. Dotted line is a guide for the eye.

librium. It is indeed these NP's adsorbed on the PAM chains which are reinforcing the polymeric array.

These attractive interactions existing between iron oxide nanoparticles and the PAM chains in the ferrogels are ascribed to hydrogen bonds between the carbonyl groups of the polymer (each monomer bearing a primary amide function) and hydroxyl functions at the iron oxide surface, either $\mathrm{Fe}-\mathrm{OH}$ directly at the solid surface or hydroxyl end-groups from the citrate ligands coating the nanoparticles. The hypothesis of $\mathrm{H}$-bonds formation between PAM and many oxides was proposed long ago [13]. It was evidenced by different experimental techniques $[14,15]$. Such H-bonds were also invoked in the kinetics of adsorption of PAM onto the haematite ( $\alpha$ $\left.\mathrm{Fe}_{2} \mathrm{O}_{3}\right)$ surface, which has a chemical reactivity close to maghemite $\left(\gamma-\mathrm{Fe}_{2} \mathrm{O}_{3}\right)$ [16]. This NP's - PAM adsorption is also evidenced by AFM probing as attested by Figure 6 which shows the formation of NP's necklaces along PAM chains [17].

\subsection{Experimental results at $R_{S} \sim 1$ and $R_{\Pi}<1$}

In these conditions, the swelling ratios of the ferrogels remain constant : $G_{\text {equil }}^{F G}=G_{\text {synth }}^{F G}$ and the release ratio $R$ equals either 0 for $\Phi_{\text {synth }}<4 \%$ or almost 1 for $\Phi_{\text {synth }}>4 \%$ (see series I in Table 1).

The SANS probing at swelling equilibrium shows an up-turn of $S(q)$ at low $q$ 's marking some NP inhomogeneities in the ferrogel (see Figure 3). This up-turn,

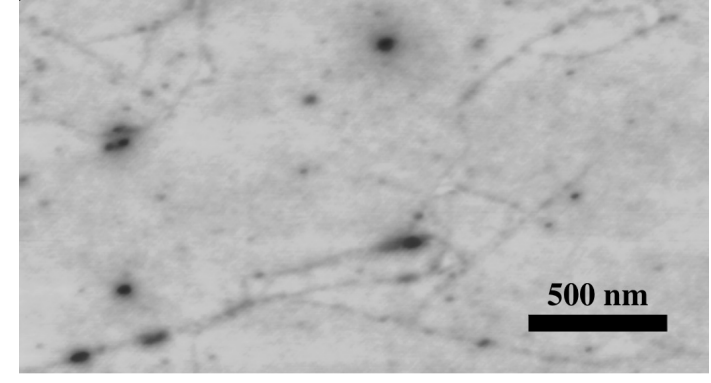

Figure 6: AFM picture of a dilute system of PAM chains and citratecoated maghemite NP's at $[\text { cit }]_{\text {free }}=810^{-3}$ mol.L ${ }^{-1}$ deposited on a mica substrate.

which is more and more marked as $\Phi_{\text {synth }}$ increases, is associated to a bump at intermediate $q_{\max }$ pointing the most-probable interparticle distance $\delta_{m p}$ in the FG. For example in the ferrogel at $C L=2 \%$ of Figure $3, q_{\max }$ equals $0.038 \AA^{-1}$ meaning $\delta_{m p} \sim 165 \AA$, which is much larger than the value $\xi_{\text {equil }}$ that would be obtained in a homogeneous hydrogel. As well it is smaller than the mean interparticle distance deduced from $\Phi_{\text {equil }}$ (but of the same order as the one deduced from $\left.\Phi_{\text {synth }}\right)$.

Both in the synthesis conditions and at swelling equilibrium, magneto-birefringence measurements show that the NPs free to rotate have a characteristic rotational time close to that of the initial MF. At swelling equilibrium, if there is no NP release, some NPs are blocked, however if the release ratio $R$ is large the majority of the NPs are blocked in rotation. All these observations converge to the picture sketched in Figure 5 for the ferrogels synthesized at $R_{S} \sim 1$. In the synthesis conditions, the polymeric network has a very heterogeneous structure with areas of tigh mesh size (excluding NPs) coexisting with pockets free of polymer and full of ML. At low $\Phi_{\text {synth }}$, the ferrogels, unable to swell in the water bath, release no NP. As $\Phi_{\text {synth }}$ becomes larger than $4 \%$, the ML-pockets becomes too large and/or too numerous, they tear up in the swelling bath, leaving behind NP-decorated walls with a NP-interspacing close to that of the previous inside-pocket ML.

\section{Results and discussion at high $[\text { cit }]_{\text {free }}$}

Let us now examine systems either synthesized at $[\text { cit }]_{\text {free }}=810^{-3}$ mol. $\mathrm{L}^{-1}$ and then swollen at equilibrium in a bath at $[\mathrm{cit}]_{\text {free }}=0.1 \mathrm{~mol} . \mathrm{L}^{-1}$, or systems both synthesized and swollen at equilibrium with $[\text { cit }]_{\text {free }}=$ $0.1 \mathrm{~mol}^{-\mathrm{L}^{-1}}$.

A first set of experiments is undertaken at $R_{S}<1$, $R_{\Pi}<1, C L=0.5 \%$ and $1 \%$, with ferrogels synthesized 


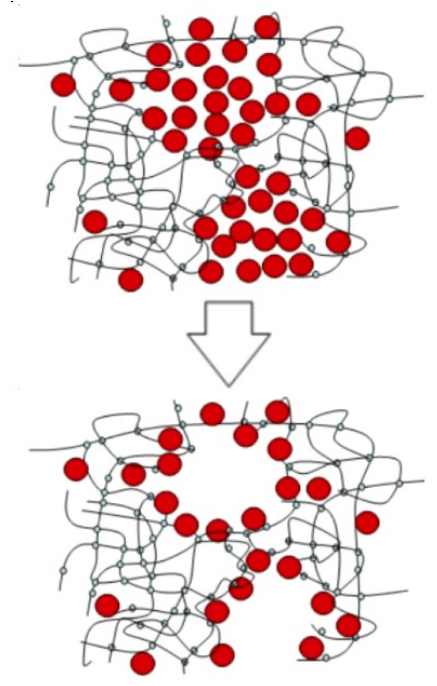

Figure 7: Picture of the heterogeneous ferrogels at $R_{S} \sim 1, R_{\Pi}<1$ (see series I of Table 1) : (left) with pockets full of ML in the synthesis conditions, (right) with empty NP-decorated pockets.

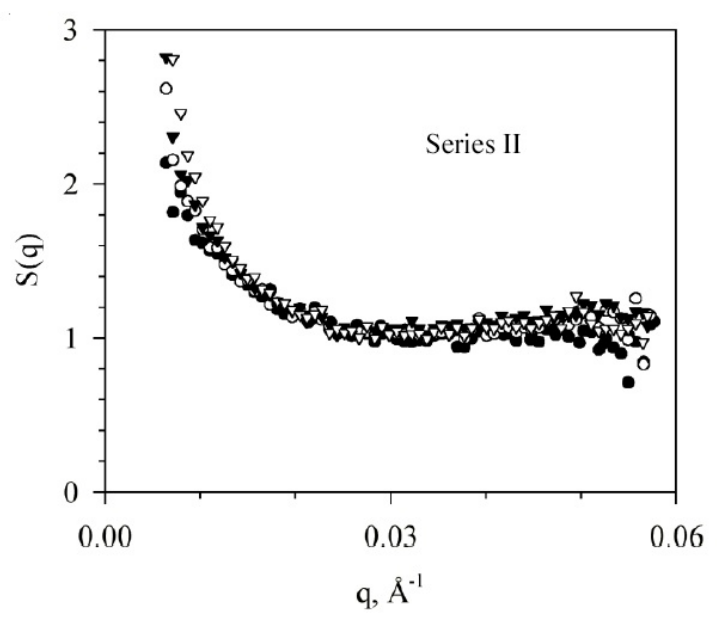

Figure 8: Structure factor $\mathrm{S}(\mathrm{q})$ of samples from series II synthesized at $[\text { cit }]_{\text {free }}=810^{-3}$ mol.L and swollen at $[\text { cit }]_{\text {free }}=0.1 \mathrm{~mol} . \mathrm{L}^{-1}$; Symbols $\Phi_{\text {equil }}^{I I}=0.14 \%$ (closed circles), $0.2 \%$ (open circles), $0.54 \%$ (closed triangles) and $0.67 \%$ (open triangles).

\begin{tabular}{|c|c|c|c|}
\hline$\Phi_{\text {synth }}(\%)$ & $\Phi_{\text {equil }}^{I}(\%)$ & $\Phi_{\text {equil }}^{I I}(\%)$ & $\Phi_{\text {equil }}^{I I I}(\%)$ \\
\hline 1 & $0.97(*)$ & 0.14 & 0.08 \\
\hline 3 & $2.95(*)$ & 0.2 & 0.38 \\
\hline 5 & 0.76 & 0.54 & 0.54 \\
\hline 7 & 0.82 & 0.67 & 0.71 \\
\hline
\end{tabular}

Table 1: Experiments in heterogeneous ferrogels at $R_{S} \sim 1, R_{\Pi}<$ 1 and $C L=2 \%, \Phi_{\text {synth }}$ and $\Phi_{\text {equil }}$ for the three series I, II and III described in the text. (*) Samples without any NP's release, thus with unteared ML-pockets. at $[\text { cit }]_{\text {free }}=810^{-3}$ mol. $\mathrm{L}^{-1}$ and swollen at equilibrium at 0.1 mol. $\mathrm{L}^{-1}$. Only small modifications are detected in the measurements of $R, G_{\text {equil }}$ and $\Phi_{\text {equil }}$. The release ratio $R$ and the swelling ratio $G_{\text {equil }}$ are both $30 \%$ larger, which lets suspect that the interaction between NP's and polymeric chains is slightly decreased and that free citrate enters in competition with NP's in the PAM adsorption process.

A second set of measurements is presented in Table I. These experiments are performed at $R_{S} \sim 1, R_{\Pi}<1$ and $C L=2 \%$, series I correspond to samples both synthesized and swollen at $[\text { cit }]_{\text {free }}=810^{-3}$ mol.L ${ }^{-1}$ (as those of section 3-2), series II to samples synthesized at $[\text { cit }]_{\text {free }}=810^{-3}$ mol.L and swollen at $[\text { cit }]_{\text {free }}=$ 0.1 mol.L ${ }^{-1}$, serie III to samples both synthesized and swollen at $[\text { cit }]_{\text {free }}=0.1$ mol. $\mathrm{L}^{-1}$. The only visible effect of the large concentration of citrate either in synthesis conditions or in swelling conditions is to provoke a large NP release at $\Phi_{\text {synth }}=1 \%$ and 3\%, tearing up any heterogeneous ML pocket in these ferrogels. As a summary of Table I, we can say that either there is no release and no swelling in the swelling bath then $\Phi_{\text {equil }} \sim \Phi_{\text {synth }}$ and some ML pockets are remaining in the ferrogel (samples marked with $\left(^{*}\right)$ in table 1), or the ML pockets tear up in the swelling bath, then only few NP's remain in the gel and $\Phi_{\text {equil }} \sim \Phi_{\text {synth }} / 10$.

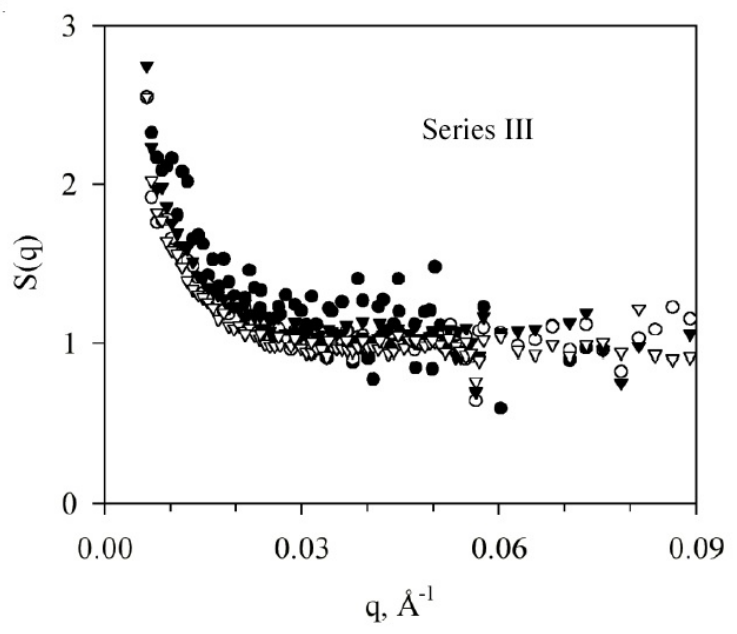

Figure 9: Structure factor $S(q)$ of samples from series III both synthesized and swollen at $[\text { cit }]_{\text {free }}=0.1$ mol.L ${ }^{-1} ;$ Symbols $\Phi_{\text {equil }}^{I I}=0.08 \%$ (closed circles), $0.38 \%$ (open circles), $0.54 \%$ (closed triangles) and $0.71 \%$ (open triangles).

Small Angle Neutron Scattering experiments have been performed on these samples. The NP structure factor S(q) of the sample from Series I at $\Phi_{\text {equil }}^{I}=0.76 \%$ 
was presented previously in Figure 3. Those of series II and III are plotted in Figures 7 and 8. They are completely similar for both series whatever is $\Phi_{\text {synth }}$. They reveal an inhomogeneous distribution of NP's, without any correlation peak associated to a most probable interparticle distance, contrary to what is observed in Figure 3. These $\mathrm{S}(\mathrm{q})$ are on the contrary very close to the structure factor in the pure ML at $[\text { cit }]_{\text {free }}=0.1$ mol. $\mathrm{L}^{-1}$, where the interparticle interaction is attractive. We should note that they are independent on the value of $[\text { cit }]_{\text {free }}$ in the synthesis conditions, on the contrary it is the value of $[\mathrm{cit}]_{\text {free }}$ in the swelling bath which determines the final structure.

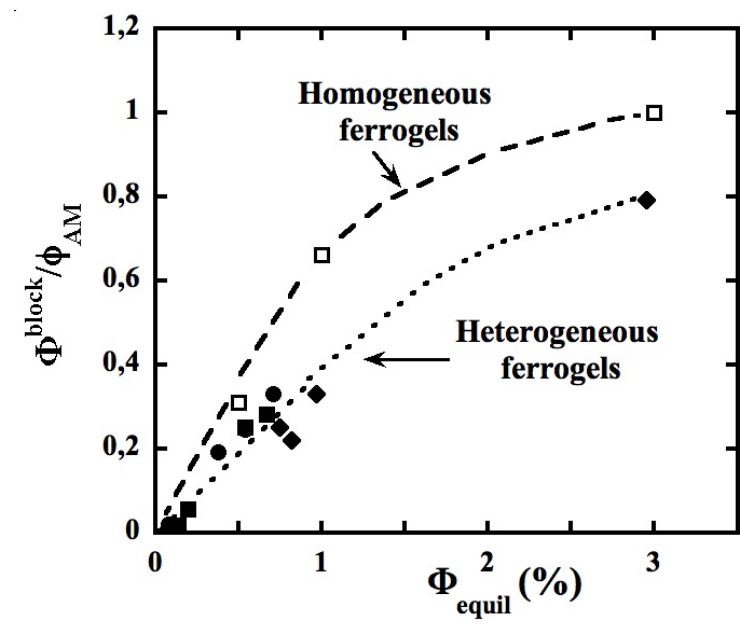

Figure 10: Volume ratio of blocked NP's with respect to polymer at swelling equilibrium versus NP's volume fraction at swelling equilibrium $\Phi_{\text {equil }}$. Comparison between : - homogeneously decorated networks at $C L=0.5 \%, R_{S}<1$ and $R_{\Pi}<1$ (open squares : $[\text { cit }]_{\text {free }}$ $=810^{-3}$ mol. $\mathrm{L}^{-1}$ both in synthesis conditions and at swelling equilibrium) and - inhomogeneous networks at $C L=2 \%, R_{S} \sim 1$ and $R_{\Pi}<1$ (closed symbols samples of Table I, diamonds : series I, squares : series II and circles : series III). Dashed and dotted lines are guides for the eye.

A probing of these samples by relaxation of magnetooptical birefringence is also performed, allowing to deduce the proportion of blocked NP's in the various ferrogels. Figure 10 compares $\Phi^{\text {block }} / \phi_{A M}$, the volume ratio of blocked NP's with respect to polymer in the swollen ferrogels as a function of $\Phi_{\text {equil }}$ for homogeneously decorated networks and for the three series of heterogeneous networks of Table 1. There is a clear shift in NP's adsorption between the homogeneously decorated networks and the heterogeneous ones. However in the heterogeneous case, there is no visible effect of the value of $[c i t]_{\text {free }}$ on $\Phi^{\text {block}} / \phi_{A M}$, all the samples of Table 1 join together in the same master curve at $\Phi_{\text {equil }}<1 \%$. The value of $[\text { cit }]_{\text {free }}$ only determines the low value of $\Phi_{\text {equil }}$. There are here two kinds of competitions with the process of NP's adsorption on PAM chains, the adsorption of free citrate on PAM and NP's interparticle attractions.

\section{Conclusion}

In these ferrogels synthesized at $R_{\Pi}<1$ and [cit $]_{\text {free }}$ $=810^{-3} \mathrm{~mol} . \mathrm{L}^{-1}$, there is a coexistence of free-to-rotate NPs and of adsorbed ones on the PAM network. It is present both in the inhomogeneous gels $\left(R_{S} \sim 1\right)$ and in the homogeneous ones $\left(R_{S}<1\right)$ where it explains the observed reinforcing of the polymeric network. This adsorption, presumably due to H-bonding between NP's and PAM chains enters in competition with other processes at larger $[\text { cit }]_{\text {free }}$ values, the relevant value for the final structure being that of the swelling bath.

\section{Acknowledgments}

We thank D. Talbot for the ML synthesis and for the chemical titration of iron in the ferrogels. This work was supported by CONACYT Mexico.

\section{References}

[1] Z. Varga, G. Filipcsei and M. Zrinyi Polymer 47 (2006) 227.

[2] S. Abramchuk, E. Kramarenko, G. Stepanov, L.V. Nikitin, G. Filipcsei, A.R. Khkhlov and M. Zrinyi Polym. Adv. Technol. 18 (2007) 883.

[3] J. Oberdisse, P. Hine and W. Pyckhout-Hintzen Soft Matter 3 (2007) 476.

[4] J. Jestin, F. Cousin, I. Dubois, C. Ménager, R. Schweins, J. Oberdisse and F. Boué Adv. Mater. 20 (2008) 2533.

[5] A. V. Teixeira, I. Morfin, F. Ehrburger-Dolle, C. Rochas, E. Geissler, P. Licinio and P. Panine Phys. Rev. E 67 (2003) 021504.

[6] L. Benguigui and F. Boué Eur. Phys. J. B 11 (1999) 439.

[7] F. Cousin, E. Dubois and V. Cabuil Phys. Rev. E 68 (2003) 021405 .

[8] R. Massart C. R. Acad. Sci. Paris 291 (1980) 1.

[9] J.A. Galicia, O. Sandre, F. Cousin, D. Guemghar, C. Ménager and V. Cabuil J. Phys.: Condens. Matter 15 (2003) S1379.

[10] J.A. Galicia, F. Cousin, E. Dubois, O. Sandre, V. Cabuil and R. Perzynski Soft Matt. 5 (2009) 2614.

[11] M. Avdeev, E. Dubois, G. Mériguet, E. Wandersman, V.M. Garamus, A.V. Feoktystov and R. Perzynski J. Appl. Cryst. 42 (2009) 1009.

[12] E. Hasmonay, A. Bée, J.C. Bacri and R. Perzynski J. Phys. Chem. B 103 (1999) 6421.

[13] O. Griot and J. A. Kitchener Trans. Faraday. Soc. 61 (1965) 1026.

[14] M. J. McGuire, J. Addai-Mensah, K. E. Bremmell J. Coll. Inter. Sci 299 (2006) 547.

[15] S. Chibowski and M. Wisniewska Coll. Surf. A 208 (2002) 131.

[16] A. K. Bajpai and S. K. Bajpai Coll. Surf. A 101 (1995) 21.

[17] D. El Kharrat, O. Sandre, P. Licinio and R. Perzynski Slow Dynamics in Complex Systems - Book series AIP Proceedings $\mathbf{7 0 8}$ (2004) 122. 